\title{
La première Ecole laïque d'infirmiers et d'infirmières de Genève (1896-1901)
}

par Joëlle Droux

\section{RÉSUMÉ}

La création de la première Ecole laïque d'infirmières et d'infirmiers du canton de Genève (1896-1901) est indissociable du contexte socio-politique et religieux mouvementé dans lequel elle a fait son apparition, et auquel elle est redevable de la plupart de ses caractéristiques : issue d'un débat polémique articulé autour de la difficile affirmation de la laïcité de l'Etat et des services publics au tournant du siècle, elle devait susciter de violentes réactions de rejet chez certains partis politiques, professionnels de la santé, ou sympathisants religieux. Elaborée pour répondre à l'introduction des diaconesses de Berne à l'hôpital de Genève en 1891 par la formation locale d'un personnel soignant laïc de qualité, elle n'allait rencontrer auprès des Genevois qu'un accueil frileux. A travers les débats houleux que provoqua cette école, c'est tout à la fois le statut professionnel des soignants, leur position floue dans la hiérarchie hospitalière ainsi que les représentations attachées à leur fonction qui se trouvent mis en jeu.

C'est à la fin du XIX ${ }^{e}$ siècle que le canton de Genève devait connaître la première tentative d'institutionnalisation de formation de ses services infirmiers, grâce à la création d'une Ecole laïque d'infirmiers et d'infirmières, implantée à l'Hôpital Cantonal dès 1896. Cette institution, demeurée longtemps ignorée dans l'histoire de la santé publique du canton en raison de sa brièveté (elle disparaît dès 1901), méritait cependant qu'on lui consacrât quelques recherches. Le présent article a pour but d'éclaircir les conditions dans lesquelles cet établissement a été créé, puis démembré peu après, l'ensemble de ces circonstances révélant les tensions multiformes au sein desquelles les politiques de santé de nos sociétés contemporaines ont été élaborées.

\section{Contexte socio-politique}

Avant d'aborder l'histoire proprement dite de cette école, il convient de s'arrêter sur le contexte socio-politique dans lequel elle a fait son apparition, 
afin d'examiner les éléments ambiants qui allaient jouer un rôle prépondérant dans sa constitution, et dans les caractéristiques de sa courte vie.

\section{Eléments d'ordre politique}

Sans entrer dans le détail, on peut schématiser la vie politique genevoise du dernier quart du XIX ${ }^{\mathrm{e}}$ en la dépeignant comme un affrontement constant entre radicaux et conservateurs ${ }^{1}$. Ces deux partis se livrent à tout propos à d'incessantes polémiques, et chaque échéance électorale les voit s'opposer avec une infatigable virulence. En 1895, les conservateurs sont à la tête du gouvernement, menés par leur leader Gustave Ador; au pouvoir depuis 1889, ce fameux «Régime Ador» présentait toutes les caractéristiques d'un conservatisme frileux: préoccupé avant tout de l'équilibre du budget de l'Etat, ce gouvernement peu porté à l'innovation réduisit au strict minimum l'ensemble des dépenses de l'Etat. Cette gestion excessivement pondérée confère à la période, aux dires mêmes des contemporains, une impression générale d'immobilisme et de stagnation.

De l'autre côté, le parti radical et son brillant leader Georges Favon prônent une conception plus dynamique et sociale de l'action étatique. Le programme des radicaux, nettement marqué par ses préoccupations socialisantes, s'oppose non sans une nuance de démagogie à celui des conservateurs, taxé de «politique d'administrateurs et non de constructeurs» ${ }^{2}$. Au premier rang de ce programme figure la création d'institutions utiles à la collectivité, et susceptibles d'améliorer les conditions de vie des classes sociales défavorisées. L'ensemble des projets de loi radicaux de cette fin de siècle témoigne de cette optique largement socialisante, portant pour une large part l'empreinte personnelle de Favon. Par ailleurs, c'est en grande partie grâce à une campagne axée sur l'absence de créations sociales du Régime Ador que les radicaux gagneront l'adhésion de la population genevoise, qui les portera au pouvoir en 1897.

\section{Eléments religieux}

L'histoire religieuse tumultueuse de Genève, canton biconfessionnel, en cette fin de siècle, ne doit pas non plus être négligée. Le dernier quart du XIX ${ }^{e}$ est en effet une période d'affrontements confessionnels violents, qui menacèrent 
plus d'une fois la paix sociale du canton. Il est impossible de citer ici tous les conflits illustrant cette phase du «Kulturkampf» genevois; mentionnons cependant deux de ces épisodes, parmi les plus significatifs et les plus déterminants pour l'histoire ultérieure du canton. En 1872-73, le débat fratricide portant sur l'organisation du culte catholique ${ }^{3}$, décidé par le fameux gouvernement radical d'Antoine Carteret, allait déboucher dès 1875 sur la suppression des corporations religieuses ${ }^{4}$. Cet événement frappa de plein fouet les catholiques, et allait dresser les uns contre les autres pour des dizaines d'années les tenants des deux religions. D'autre part, les campagnes menées durant cette période, à plusieurs reprises, mais sans succès, en faveur de la suppression du budget des cultes, amenaient la discussion sur le thème, extrêmement mobilisateur et controversé, de la laïcisation de l'Etat. Ces débats eurent pour effet de radicaliser encore un peu plus des positions déjà rigides des protestants, premières «victimes» potentielles d'une telle mesure.

En bref, le contexte religieux fait peser sur toutes les discussions politiques de la période une puissante contrainte, rendant la tâche des gouvernants plus que délicate. Ceux-ci, quelle que soit leur couleur politique, sont assis sur une poudrière que le moindre faux pas peut transformer, au regard des sensibilités chatouilleuses des catholiques, des protestants, ou des libre penseurs, en une véritable bombe. Tous les éléments sont donc réunis, en cette fin de siècle, pour créer, à propos de n'importe quel sujet touchant au sentiment religieux, les conditions d'une polémique ravageuse, attisée par les intérêts sous-jacents de partis politiques toujours prompts à la controverse.

\section{L'Ecole lä̈que d'infirmiers et d'infirmières de Genève: une création polémique et éphémère}

L'introduction des diaconesses à l'hôpital de Genève : l'étincelle qui mit le feu aux poudres

L'Ecole laïque d'infirmiers de Genève est justement née d'une de ces polémiques. Tout commença par une décision lourde de conséquences, prise par la direction de l'Hôpital Cantonal de Genève. Le 13 avril 1891, celle-ci décidait, sur la proposition d'un médecin de l'établissement, le bernois Alcide Jentzer, de réorganiser les services infirmiers en remplaçant les soignants laïques par des diaconesses de Berne ${ }^{5}$. L'affaire est bientôt conclue avec la direction bernoise des diaconesses, et le 2 octobre 1891, une vingtaine de celles-ci font 
leur entrée à l'hôpital, dans le service de chirurgie. Elles seront suivies quelques mois plus tard par 15 de leurs consœurs, destinées au service de médecine ${ }^{6}$. Immédiatement, la polémique éclate avec violence: le 10 octobre 1891, le leader radical G. Favon, libre penseur convaincu, prend à partie le gouvernement Ador, autorité de tutelle de la direction de l'hôpital, et réclame devant le Grand Conseil le renvoi des diaconesses ${ }^{7}$. Ses arguments soulignent avec force l'incongruité d'une telle décision symbolique en regard de l'atmosphère confessionnelle tendue du canton: le risque de propagande religieuse de la part des diaconesses porterait, selon lui, une atteinte grave à la liberté de pensée des malades catholiques ou athées. D’autre part, et les radicaux appuient particulièrement sur ce point, on risque fort de susciter en retour les revendications des catholiques, désireux de rétablir pour soigner leurs coreligionnaires ces corporations religieuses supprimées, non sans une âpre opposition, en 1875. Ce dont Favon menace le Régime Ador, ce n'est rien moins que d'une nouvelle guerre de religion.

Pour le leader radical, la seule solution est de rétablir l'équilibre confessionnel le plus strict, en ne favorisant aucun des cultes, c'est-à-dire en restaurant la laïcité pure et simple du service soignant; au bout du compte, c'est la nécessité d'avoir un Etat lä̈ que Favon réaffirme haut et fort, évoquant à nouveau, implicitement, la douloureuse question de la séparation de l'Eglise et de l'Etat. Le Genevois, organe de presse du parti radical, ne manque pas de se faire l'écho des inquiétudes de son leader:

\begin{abstract}
«N'avons-nous pas au contraire pour règle absolue, dans nos services publics, de ne nous occuper en rien de ce que les citoyens pensent sur la religion? ... Demandons-nous au maître d'école, au gendarme, à nos députés, à nos conseillers d'Etat, à qui nous confions la destinée du pays et de nos plus grands intérêts, s'ils ont ou non des convictions religieuses? Où irionsnous si nous agissions ainsi? Comme l'esprit religieux a pour élément la foi et se traduit par des croyances précises, par des formules, par des dogmes, nous aboutirions droit à Calvin ou au pape, peut-être à tous les deux.» ${ }^{8}$
\end{abstract}

Et, dans un premier temps, les événements semblent leur donner raison. De toutes parts, les controverses s'élèvent. Par l'intermédiaire des infirmiers laïcs de l'hôpital qui, menacés de chômage, manifestent à tout propos leur hostilité aux diaconesses:

«On se plaint beaucoup de la nourriture que les diaconesses servent aux employés laïes, relate Le Genevois. Evidemment, ceux-ci sont condamnés à disparaître les uns après les autres, c'est le plan. Dans un an, on ne parlera plus à l'hôpital cantonal que le patois de l'Emmenthal...».9 
Par l'intermédiaire de la presse politique, surtout, qui relaye les plaintes des malades catholiques qui se disent «saturés de prières et d'invocations qui n'ont pas de limite déterminée» ${ }^{10}$. Sans compter que l'acclimatation des Bernoises ne se fait pas sans mal, et provoque le déclenchement d'épisodes tragicomiques dont les journalistes se délectent sans scrupules ${ }^{11}$. Malgré tout, la direction de l'hôpital tint bon, et refusa de renvoyer les diaconesses, affirmant que leurs qualifications paramédicales les rendaient indispensables aux services hospitaliers genevois.

\section{L'Ecole, arme de guerre des radicaux: une création polémique}

C'est alors que le parti radical va mettre sur pied son «arme de guerre antidiaconesses» ${ }^{12}$, sous la forme d'un projet de loi créant à Genève une Ecole laïque d'infirmiers et d'infirmières. Réutilisant à son profit l'argument de la qualification, Georges Favon, auteur du texte, affirme avec vigueur que la seule façon d'allier laïcité et capacités soignantes, c'est de donner aux soignants laïcs un réel enseignement para-médical, en instituant pour eux une Ecole:

«Lorsqu'on a voulu, on a eu, partout, dans les écoles et dans les hôpitaux, un personnel laïque à la hauteur; seulement, cela coûte un peu plus de peine et plus cher... [Il faut] créer une école d'infirmiers, de gardes-malades, donner plus d'instruction à ses serviteurs et les payer davantage, afin de les avoir comme on les a quand on les paie: capables, dévoués, propres et actifs». ${ }^{13}$

A court terme, cette innovation devait permettre d'apaiser les querelles religieuses et de restaurer à Genève la concorde entre les citoyens. A moyen terme, les radicaux escomptaient, grâce aux longs débats publics qui allaient suivre, mettre à profit leur image de défenseur de la laicité et de la liberté de pensée à des fins électorales, aux dépens du gouvernement conservateur, dont la bienveillance à l'égard des diaconesses devenait chaque jour plus évidente ${ }^{14}$. A long terme, cette solution permettait de former puis de réintégrer un personnel laïc de qualité dans les services infirmiers de l'hôpital, et donc de renvoyer chez elles les diaconesses bernoises. On faisait ainsi d'une pierre deux coups: on créait un débouché nouveau pour les travailleurs genevois, en leur ouvrant une profession à part entière, dotée d'une formation de qualité et gratifiée de salaires avantageux, articulant ainsi avec bonheur la lettre du projet à l'esprit résolument socialisant du programme radical; d'autre part, on se débarrassait d'une ingérence bernoise mal ressentie par des mentalités genevoises au chauvinisme tâtillon. 
Dès le début, donc, cette école avait été conçue dans une optique largement polémique; elle porta durant toute sa courte vie les stigmates de cet enfantement lourd de controverses et de faux-semblants démagogiques.

\section{L'Ecole, une création éphémère}

Il est impossible de résumer, même brièvement, la teneur des débats suscités alors à Genève à propos du projet Favon: ceux-ci s'étendent sur près de deux cents pages des volumes des Mémoriaux du Grand Conseil, et se déploient sur plus de cinq ans: de 1891, date de l'entrée des diaconesses, au 8 novembre 1893, date de présentation officielle du projet devant le Grand Conseil, et jusqu'au 16 mai 1896, date à laquelle le projet fut définitivement adopté. Il aura fallu passer par la nomination d'une commission, par de multiples enquêtes auprès des médecins et chefs de cliniques genevois, par des engagements épiques entre les députés, le tout couronné par des campagnes de presse acharnées, pour voir le projet aboutir et se concrétiser: votée en 1896 par 44 voix, contre 16 non et 14 abstentions ${ }^{15}$, l'Ecole fut mise en œuvre dès la même année. Abritée dans les locaux de l'hôpital cantonal, elle offrait sur 40 semaines un enseignement théorique de 18 heures par semaine, assuré conjointement par des médecins, des assistants et des pharmaciens. La formation était complétée par un stage accompli dans les services de l'hôpital, et se concluait par un examen théorique et pratique, sanctionné par un certificat d'études ${ }^{16}$.

L'école ne devait durer que 5 ans. En effet, après un débat sommaire, et au milieu, semble-t-il, de l'indifférence générale, le Grand Conseil devait mettre un terme à cette expérience le 22 mai $1901^{17}$. Les promoteurs du projet durent se rendre à l'évidence: l'école était un échec cuisant. En 5 ans, seuls 22 élèves s'étaient inscrits pour suivre ses cours; alors qu'elle avait été organisée pour recevoir 16 élèves par an, le nombre d'inscriptions annuelles n'avait jamais dépassé 6 candidats ${ }^{18}$. D'un autre côté, l'Etat genevois avait déjà dépensé plus de 17000 francs pour assurer la couverture des frais suscités par l'établissement: dans de telles conditions, la situation n'était pas viable. L'école dut fermer ses portes.

L'intérêt de cette école ne réside évidemment pas dans sa durée, mais dans la violence et la multiplicité des réactions qu'elle a fait naître. Les polémiques ardentes qui ont conduit à sa création représentent pour l'histoire sociale de la médecine un moment privilégié d'observation et d'analyse. En effet, les discours prononcés pour ou contre cette fondation nous permet- 
tent d'examiner, avec un luxe de détails significatifs, quelle image la population genevoise et ses responsables politiques se faisaient de la profession de soignant en cette fin de $\mathrm{XIX}^{\mathrm{e}}$ siècle. A travers ces virulents échanges d'arguments, nous nous trouverons au cœur des mentalités; nous entrons de plain-pied dans l'univers sibyllin des représentations liées au domaine de la santé et de sa préservation, du soin des corps et du soin des âmes. Plus précisément, la question de l'évolution contemporaine des professions de santé apparaît comme étant au cœur d'un écheveau de significations qu'il nous appartient de démêler: elle met en cause plusieurs niveaux d'interprétation et de représentations, qui aujourd'hui nous échappent en partie, mais qui à l'époque étaient vigoureusement ressenties. A cet égard, l'ampleur inhabituelle des débats liés à ce projet sur la scène politique, les trésors de combativité qui y furent déployés, de même que la mobilisation de la presse et de la rue, témoignent à leur manière de l'impact démesuré de cet événement, en apparence anodin. Comment expliquer notamment la détermination farouche des opposants, alors même que ce projet semblait aller dans le sens d'une plus grande modernité des services de santé genevois? Et comment expliquer l'échec, si rapide, de cette école, et l'indifférence avec laquelle l'accueillit la population laborieuse de Genève, pour qui pourtant elle avait été conçue? Pour répondre à ces questions, il est nécessaire de se pencher sur l'examen du niveau médian d'appréhension de la réalité que constitue le discours. Au travers d'une analyse de ce type, on peut avancer que si l'Ecole laïque d'infirmiers et d'infirmières de Genève s'est heurtée à l'opposition d'une partie de l'opinion publique, des milieux médicaux, des hommes politiques et de la presse locale, c'est parce qu'elle imposait à Genève un modèle des soins et du soignant en tous points étranger à la représentation dominante associée à ceux-ci à la fin du siècle dernier.

Il est nécessaire de sélectionner, étant donné l'abondance des échanges rhétoriques développés à cette occasion, quelques-uns des principaux éléments sur lesquels les mentalités genevoises n'étaient pas disposées à transiger dans leur représentation idéale de l'acte et de la personne soignante.

\section{Eléments liés à la nationalité du soignant}

La récurrence des arguments d'ordre nationaliste est une des caractéristiques dominantes de ces débats: Favon et les radicaux considèrent l'avènement de cette école comme un excellent moyen d'embaucher des Genevois 
peu fortunés, en réhabilitant pour eux une profession jusqu'alors méconnue et déconsidérée:

«Si nous fournissions à des Genevois, par un enseignement approprié, les moyens de gagner leur vie dans une carrière quelconque, nous trouverions actuellement à Genève des hommes aussi capables de remplir leur mandat que ceux qui peuvent nous venir de l'étranger». ${ }^{19}$

En outre, le recrutement de Genevois dans les services hospitaliers permettrait de débarrasser l'hôpital des diaconesses bernoises, dont les radicaux soulignent avec une ironie appuyée et quelque peu xénophobe qu' «après trois ans de séjour chez nous, dans un service public, [elles] ne savent pas un mot de français» ${ }^{20}$. Mais, alors même que Favon manipule, non sans arrièrepensée, des arguments nationalistes pour appuyer son projet, ce sont des mobiles de même nature que lui opposent ses adversaires, à la tête desquels Gustave Ador justifie ainsi ses réticences:

«Je crains que notre caractère national ne soit pas tel que nos concitoyens pussent remplir avec succès les fonctions d'infirmiers et d'infirmières... A notre hôpital cantonal, à plusieurs reprises, on a tâché d'avoir de bons infirmiers et de bonnes infirmières de nationalité genevoise... et l'on a rarement réussi... Très rares sont les Genevois qui acceptent cette position, qui n'est pas très brillante du point de vue des salaires. Les jeunes filles, notamment, préfèrent en général à la position d'infirmière celle de domestique, de couturière, ou de modiste». ${ }^{21}$

L'expérience éphémère de l'école allait se charger de confirmer le pressentiment défavorable du leader conservateur: sur les 22 élèves que recrutera l'école, 4 seulement seront genevois, et parmi eux aucune femme. La résistance active manifestée contre l'école par les partisans de Gustave Ador se concrétisa donc effectivement sur le terrain par une résistance passive des Genevois, dont la désaffection à l'égard de la profession de soignant semblait décidément bien enracinée. Rechercher les causes d'une telle répugnance nous entraînerait trop loin dans le cadre de cette étude, mais nécessiterait à l'évidence une plongée encore plus profonde dans les mentalités des périodes antérieures.

\section{Caractères liés à la religion}

Alors que Favon et ses amis visaient explicitement, en rétablissant la laïcité des soins infirmiers de l'hôpital, à acheminer Genève sur la voie de la séparation irrémédiable de l'Eglise et de l'Etat, l'opposition religieuse se manifeste avec hostilité. Curieusement, catholiques et protestants, malgré 
leurs antagonismes séculaires, allaient se retrouver côte à côte pour défendre une conception des soins infirmiers fondamentalement religieuse. Selon eux, seule la foi peut insuffler aux soignants un dévouement et un désintéressement irréprochables, parce qu'inspirés par l'amour chrétien du prochain. A l'inverse, on craint qu'un personnel laïc, uniquement motivé par l'appât du gain, ne se montre cupide, corrupteur, en un mot immoral. De plus, les tenants des deux religions soulignent de concert la double utilité du soignant religieux, seul capable d'allier soins physiques et consolation spirituelle, deux versants d'une seule et même réalité de souffrance, et d'apporter ainsi au malade, selon le mot de G. Ador «le sourire qui guérit en même temps que le remède du médecin» :

«Je suis de ceux qui croient qu'en matière de soins à donner aux malades, il faut l'inspiration d'un sentiment plus élevé que le simple appât d'un salaire; je crois qu'il faut le dévouement, l'esprit de charité, en un mot des convictions d'un principe supérieur au terre à terre de la laïcité et du pouvoir civil». ${ }^{22}$

L'omniprésence de ce type d'argument dans les débats montre bien qu'à cette époque, l’image du soignant est encore imprégnée de connotations caritatives et philanthropiques essentiellement religieuses, révélant par là même l'incontournable certitude que la maladie, loin de n'être qu'expérience physique est aussi, et surtout, une épreuve morale et psychologique que la réponse scientifique ne peut prétendre résoudre à elle seule. Reste que, si l'on admet volontiers l'existence du médecin ou du savant humanistes, œuvrant pour le bien de leurs semblables, on dénie à l'infirmier laïc toute possibilité d'accéder à une telle hauteur de sentiment. Si les capacités des médecins se mesurent à l'aune de leur savoir scientifique, celles des soignants «subalternes» demeurent en étroite dépendance avec leurs seules convictions religieuses. Sur ce terrain, catholiques et protestants se rejoignent donc, se faisant les avocats des diaconesses pour les uns, tandis que s'agite en filigrane, dans la pensée des autres, l'attachement aux congrégations soignantes expulsées en 1875.

\section{Eléments liés à la formation}

Sans aucun doute cette distinction est-elle très directement liée à la place qu'occupe le soignant dans la cosmologie sociale, ainsi qu'à son statut socioprofessionnel entaché de la macule du subalterne. En effet, l'argument suivant, avancé par les opposants au projet de l'école, concerne la nature et 
l'étendue de la formation envisagée. Sur cette question, on assiste au regroupement de grands patrons des cliniques et de certains députés conservateurs, fondamentalement hostiles à l'ambition éducationnelle du texte. Les uns, par la voix d'un conseiller d'Etat, A. Richard, contestent en termes révélateurs l'excès des cours théoriques contenus dans ce programme:

«Je crois qu'il n'est pas nécessaire que des infirmiers, qui ont le rôle de domestiques que vous savez..., soient au courant de tous les phénomènes biologiques de notre existence. J'y vois même un inconvénient qui vous frappera sans doute, c'est qu'il y a toujours un grand danger à faire des demi-savants, parce que la demi-science conduit à la pédanterie, et que rien ne tarit les sources du dévouement comme le sentiment que l'on est supérieur aux autres en savoir». ${ }^{23}$

Toute aussi significative est l'opposition du docteur Julliard, dont l'avis autorisé a été sollicité par le Grand Conseil, et qui répond:

«Dussé-je scandaliser les partisans du projet, je dois avouer ne pas comprendre ce qu'on peut enseigner qui vaille en fait d'anatomie, de physiologie et d'hygiène à des gens qui n'ont d'autre préparation à cette affaire que d'avoir été dans leur enfance à l'école primaire... Non, cent fois non, je ne vois pas ce qu'un individu qui a pour toute culture intellectuelle les souvenirs lointains de son école primaire pourra bien entendre à [ces] cours. Pour moi, un enseignement comme celui-là n'est pas sérieux. Il est sans valeur et sans utilité aucune.» ${ }^{24}$

A quoi la presse radicale rétorque sans coup férir, fustigeant

«le parti pris obstiné des chefs de ces deux cliniques qui... ont montré dès le début une hostilité passionnée contre la nouvelle création, d'accord en cela avec quelques membres de la Commission administrative de l'hôpital cantonal. Routine et désir de faire voir qu'on est le maître! Il ne serait peut-être pas inutile de saisir cette occasion de rappeler à ces Messieurs que leurs privilèges ont pour limite l'intérêt général et que les cliniques, comme l'Université, sont faites pour le pays, et non le pays pour les cliniques.» ${ }^{25}$

De tels aperçus permettent de moduler l'affirmation selon laquelle les progrès médicaux et scientifiques de la fin du $\mathrm{XIX}^{\mathrm{e}}$ auraient permis à une profession de soignant de s'individualiser et de s'émanciper de la tutelle médicale: l'exemple genevois prouve, s'il en était besoin, que l'évolution historique suit rarement un tracé aussi rectiligne.

On sent bien, à la violence singulière dégagée par ces arguments, que le projet Favon touche à tout autre chose qu'un simple programme pédagogique. Nombreux sont ceux qui paraissent ressentir cette école et ses implications émancipatrices comme une remise en cause des hiérarchies médicales autant que de l'ordre social. Que se soit par des médecins menacés dans leur monopole des connaissances médicales, que ce soit par les représentants d'un 
ordre bourgeois élitiste, désireux de maintenir une distance respectueuse des distinctions hiérarchiques avec les autres classes sociales, ces débats mettent en jeu une représentation statique de la société et des statuts socio-culturels qui la structurent. L'exemple de cette première Ecole laïque d'infirmiers et d'infirmières du canton de Genève prouve ainsi, s'il en était besoin, combien l'évolution contemporaine des professions de santé a pu être autant dépendante des progrès purement scientifiques que liée au spectre des diverses représentations qui configurent les mentalités d'une société donnée à un moment donné de son histoire.

Avec cet événement furtif et largement méconnu de la création de la première Ecole läque d'infirmiers et d'infirmières du canton de Genève, nous avons la possibilité d'observer les motifs et mécanismes rarement désintéressés qui ont présidé à l'élaboration des politiques sanitaires des sociétés contemporaines. On peut en conclure que si cette école n'a pas porté ses fruits, son échec n'est sans doute pas imputable à l'insuffisance des cours, non plus qu'à la médiocrité des élèves. Son insuccès s'explique bien plutôt par le fait que la société genevoise, dans son ensemble, n'était pas prête à entériner l'image du soignant que cette institution lui renvoyait: sa laïcité sans concession, son ambition éducationnelle, sa destination populaire, son caractère masculin, enfin, provoquèrent à son égard une réaction générale de rejet. La greffe n'avait pas pris, pas plus à Genève qu'ailleurs en Europe occidentale, qui consistait à faire table rase des soignants religieux pour leur substituer un personnel laïc formé dans les écoles de l'Etat. Cette étape ne sera pas franchie à Genève avant le $\mathrm{XX}^{\mathrm{e}}$ siècle. 


\section{Notes et Références}

1 Pour toute cette partie concernant la vie politique et sociale de Genève à cette époque, on se reportera à Ruchon François, Histoire politique de la République de Genève, 1813-1907, Genève, 1953.

2 Expression citée par Ruchon, op. cit.

3 «Loi sur les corporations religieuses», du 3 février 1872, in: Recueil des lois du canton de Genève [RL], 1872, pp.45-48.

4 «Arrêté législatif portant sur la suppression des corporations religieuses», du 23 août 1875, $R L, 1875$, pp. 379-383.

5 Suss A., Aux diaconesses de la maison de Berne; notice et souvenirs à l'occasion du $25^{\circ}$ anniversaire de leur entrée à l'hôpital cantonal de Genève, Genève, 1917, p. 3 sqq.

6 Ibid., p. 5 sqq. La direction bernoise envoya également un certain nombre de diacres.

7 Mémorial des séances du Grand Conseil de Genève [MGC], 1891, p. 1341 sqq.

8 Le Genevois, 22 octobre 1891.

9 Le Genevois, 7 novembre 1891.

10 Extrait d'une pétition de plusieurs malades, expédiée à la rédaction du Genevois, et à la direction de l'hôpital, et qui se clôt par cette pointe: «Nous pensons que les diaconesses employeraient plus utilement leurs loisirs à apprendre le français afin qu'elles pussent se faire comprendre, ainsi qu'à suivre des cours de pansements, connaissances qui leur sont indispensables et qui leur font défaut». (Le Genevois, 6 novembre 1891).

11 Au cours de l'année 1895, deux accidents graves se produisirent à l'hôpital, engageant la responsabilité du personnel bernois: un malade fut notamment brûlé au cours d'un bain donné par un diacre; celui-ci, ne comprenant pas le français, aurait été incapable de comprendre les protestations du malade qui se plaignait de la chaleur excessive de l'eau. L'homme devait décéder quelques jours plus tard. Ces affaires firent grand bruit auprès de la population genevoise, et attisèrent en janvier et en septembre 1895 les polémiques les plus violentes entre Le Genevois, hostile aux diaconesses, et Le Journal de Genève, organe des conservateurs, qui leur était favorable.

12 La métaphore militariste évoquée ici n'est qu'un reflet de l'état d'esprit et de la rhétorique belliqueuse des radicaux à l'égard des diaconesses, tel qu'il s'exprime par exemple dans les colonnes du Genevois : «Il s'agit ici non de la valeur morale des diaconesses ou des Sœurs, mais de la souveraineté civile, de la laïcité de l'Etat, de sa neutralité confessionnelle, de la défense de ses prérogatives. Quand on est en guerre, regarde-t-on à la moralité et à la sincérité de ceux qui portent l'uniforme ennemi ?» (Le Genevois, 14 octobre 1891).

13 MGC, 1891, p. 1352. Le véritable projet fut présenté au Grand Conseil le 8 novembre 1893.

14 Le chef du gouvernement, G. Ador, tendait, lors d'une visite aux malades de l'hôpital, «à accentuer ses précédentes déclarations en faveur de l'introduction des diaconesses à l'hôpital et à proclamer que cette mesure avait l'appui et les sympathies du Conseil d'Etat actuel» (Le Genevois, 30 décembre 1891).

15 MGC, 21 septembre 1895, p. 1335.

16 Ibid., pp. 1336-1338, texte de la loi.

17 MGC; 1901, p. 201 sqq.

18 Ibid., p. 202.

19 Intervention de Favon, MGC, 1893, p. 1308. 
20 Extrait d'un article paru dans Le Genevois, 7 janvier 1895.

21 MGC, 1893, p. 1511.

22 Intervention de G. Ador, $M G C, 1891$, p. 1349.

23 MGC, 1893, p. 1545.

$24 \quad M G C, 1895$, p. 1307. Certains médecins adopteront une position beaucoup plus mesurée, tel le Dr Thomas, médecin à l'hôpital: «Je comprends parfaitement et jusqu'à un certain point je partage les idées qui sont à la base du projet qui vous est soumis. Mais je crois qu'avant de se lancer dans cette création, il faut bien y réfléchir et savoir en particulier si, le cas échéant, on serait assuré de recruter un personnel présentant des conditions indispensables pour exercer une profession qui, si elle est une des plus utiles, comporte des difficultés considérables... Je n'ai nulle prétention à l'infaillibilité dans l'opinion que je puis avoir sur une question difficile et délicate et qui doit être traitée sans parti pris en envisageant toujours le but suprême, c'est-à-dire la meilleure manière de soigner les malades.» (Journal de Genève, 2 juin 1895).

25 Le Genevois, 5 juin 1895. 


\section{Summary}

The first laic school of nurses, male and female, at Geneva (1896-1901)

The foundation of this school is intricately bound to the socio-political and religious context of the turn of the century. Polemics about the implementation of the laic principle in the State and in public services led to the foundation of this school, which accordingly was opposed by some political parties, by part of the healing professions and by the religious-minded. As a reaction to the appointment of sisters of the protestant nursing order of Berne at Geneva Hospital, the new school was intended to form laic nursing personnel of local origin and high quality. The idea met with a cool reception by the population of Geneva and the school lived only for a few years.

\section{Zusammenfassung}

\section{Die erste laizistische Schule für Krankenpfleger und -pflegerinnen in Genf (1896-1901)}

Die Gründung der Schule ist mit dem bewegten sozio-politischen und religiösen Kontext der Jahrhundertwende aufs engste verflochten. Polemische Auseinandersetzungen um die Durchsetzung des Laizismus im Staatswesen und in den öffentlichen Diensten haben zur Gründung der Schule geführt, die dementsprechend von bestimmten politischen Parteien, Angehörigen der Heilberufe und religiös Gesinnten bekämpft wurde. Als Reaktion auf die Berufung der Berner Diakonissen ans Genfer Spital sollte sie die Ausbildung bodenständiger, nicht religiös gebundener, qualifizierter Pflegerinnen und Pfleger gewährleisten. Die Gründung wurde von den Genfern jedoch kühl aufgenommen und hatte nur kurzen Bestand.

Joëlle Droux

Département d'histoire générale

Université de Genève

20 Rue de l'Ecole-de-Médecine

1211 Genève 4 